Kuznetsov says that his team is now working to design and develop workable nanophotonic devices for industry. "I believe that resonant dielectrics, in contrast to plasmonics, have a strong chance to reach real-world industrial applications in the very near future," he told Nature Photonics. "The key features will be very low losses and CMOS compatibility."

Despite all the high-level research into plasmonics for integrated optics and optoelectronic devices such as nanoantennas, waveguides, detectors and modulators, it seems that the application closest to fruition and commercialization in the near term may actually turn out to be a strikingly simple and visual one. The answer is pigment-free decorative labels and security marks that exploit the structural colour associated with plasmonic resonances.

Anders Kristensen from the Technical University of Denmark gave a talk entitled Nanoplasmonics for Structural Colours on Consumer Products, during which he explained that the automotive industry in particular is interested in new types of visual surfaces, which could be very robust, visually striking and easy to manufacture. Working with Levy and the Israeli firm Nanonics Imaging, the team has developed thin plastic foils that feature metallic nanostructures to provide colourful, scratch-resistant labels. In Germany, the company Louisenthal is developing similar structural colour films for use in ambient light conditions with the prerequisite that the fabrication approach must be scalable to large areas and suitable for cheap mass production. In both cases, arrays of silver or aluminium nanostructures on the scale of a few hundred nanometres in size are embedded into a plastic film.

Joel Yang from IMRE in Singapore described how plasmonics based on metal nanostructures can overcome some of the limitations of conventional colour printing such as limited resolution, the fading of dyes and misalignment errors when printing with multiple colours. In contrast, he says that a plasmonic-based approach allows you to "print colour at the diffraction limit of light" and reach a resolution as high as 100,000 dpi. He's now looking at the possibility of colour mixing using different sizes of several nanostructures within a single pixel and says that he has switched from using silver to aluminium as it is much cheaper and CMOS compatible. "We've been talking to companies about applying marks to plastic bank notes or gold coins in order to improve their appearance."

Perhaps, if these printing applications take off, plasmonics will continue to have a colourful future after all.

Oliver Graydon is at Nature Photonics, The Macmillan Building, 4 Crinan Street, London N1 9XW, UK.

e-mail:o.graydon@nature.com

\title{
PHYSICS
}

\section{Laser-guided sparks}

Electrical discharges are widely used in applications such as electric welding, micromachining and fuel ignition in combustion engines, to name just a few examples. Despite their ubiquity, the ability to control and shape the exact path of a discharge along a specific trajectory remains a significant challenge. Now, Matteo Clerici and colleagues from Canada, the UK, China, France and the USA have shown that specially shaped laser beams can perform this feat (Sci. Adv. 1, e1400111; 2015).

Three kinds of beam shapes - a standard Gaussian beam, a Bessel beam and an Airy beam - were investigated in the study. All beams were produced by an amplified Ti:sapphire laser system and sent between two wire electrodes. The beams were $10 \mathrm{~mm}$ in size (full width at half-maximum), featured an input energy of $15 \mathrm{~mJ}$ and were pulsed with a pulse duration of $50 \mathrm{fs}$.

The concept of the scheme is to use the laser pulses to ionize the air between the electrodes and create a preferred path for a discharge, which is created by applying a high voltage (nearly $15 \mathrm{kV}$ over a $5 \mathrm{~cm}$ gap) between the electrodes.

The Bessel and Airy beams were able to create well-defined discharge paths (pictured), whereas in the case of the Gaussian beam the trajectory of electric

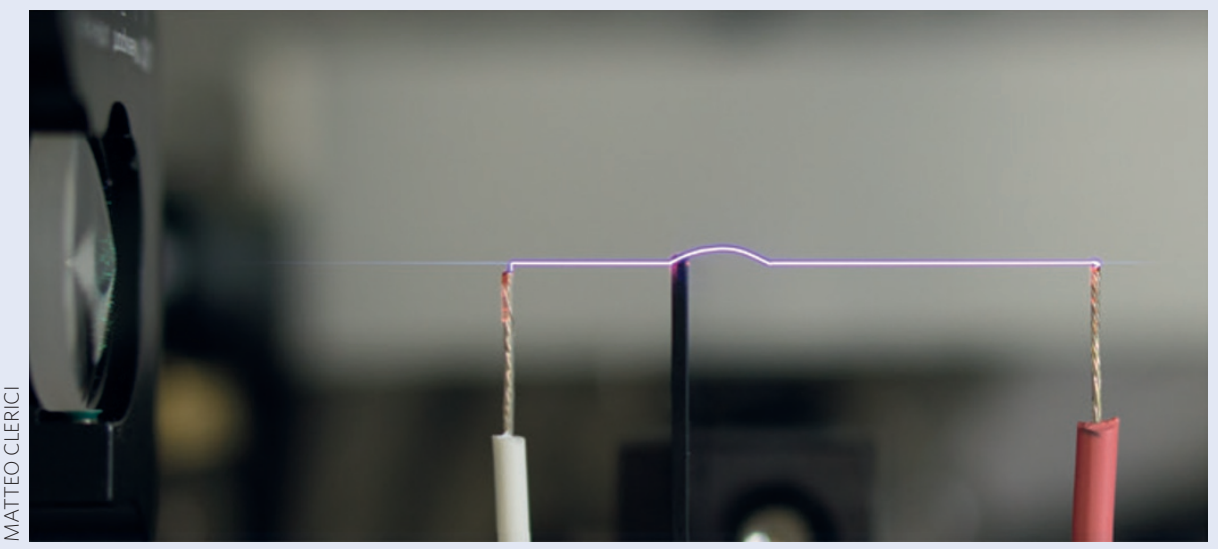

discharges is heavily disordered and effectively unpredictable.

The differences in behaviour are thought to be due to the different electric field strength of the beams. The central high-intensity peaks of the sub-diffractive Bessel beam and Airy beam were $\sim 7 \mu \mathrm{m}$ and $\sim 20 \mu \mathrm{m}$, respectively, considerably smaller than the diameter of the Gaussian optical filament $(\sim 50 \mu \mathrm{m})$. The international team further investigated the electric breakdown for the three cases and observed that the breakdown field was 3.5 and 10 times lower for the cases of the Airy and Bessel beams, respectively.

Surprisingly, the team found that laser-guided discharge could take place even when the laser beam encountered an obstacle between the electrodes (pictured). In the cases of a Bessel-type and an Airy-type propagation, the beam restored itself after the obstacle and the electric discharge occurred along an almost unaffected trajectory.

According to Matteo Clerici, one of the authors of the study, the next challenge is to find the limits of this technique. Two questions that he says need answering are: "How much can we bend a discharge and how high is the current we can transport in a controlled way?"

NORIAKI HORIUCHI 